\title{
Linkage of AD HSP and cognitive impairment to chromosome 2p: haplotype and phenotype analysis indicates variable expression and low or delayed penetrance
}

\author{
Paula C Byrne ${ }^{1}$, Stewart Webb ${ }^{2}$, Fergus M cSweeney ${ }^{1}$, Teresa B urke ${ }^{3}$, \\ $\mathrm{M}$ ichael $\mathrm{H}$ utchinson ${ }^{2}$ and Nollaig A Parfrey ${ }^{1}$ \\ D epartments of ${ }^{1}$ Pathology , ${ }^{2} \mathrm{~N}$ eurology and ${ }^{3} \mathrm{P}$ sychology, U niversity College D ublin and St Vincent's Hospital, \\ Dublin, I reland
}

\begin{abstract}
We report linkage of a family affected with autosomal dominant hereditary spastic paraparesis (HSP) and/or cognitive impairment to the HSP locus on chromosome 2p. To date all families linked to this locus have been affected with 'pure' HSP. The specific pattern of cognitive impairment in this family is characterised primarily by deficits in visuo-spatial functions. We also present genetic studies that indicate variable expression and low or delayed penetrance. We have constructed a haplotype flanked by polymorphic markers D2S400 and D2S2331 that was present in 12 individuals affected with spastic paraparesis. The severity of spasticity varied markedly among these individuals. In addition four of these individuals (aged 62-70) also had a specific form of cognitive impairment. The disease haplotype was also present in an individual (age 57) who had an identical pattern of cognitive impairment as the only sign of the disease supporting the hypothesis that spastic paraparesis and cognitive impairment are the result of variable expression of a single gene (rather than a co-incidental occurrence). Haplotype reconstruction for all participating family members revealed the presence of this disease haplotype in six individuals who had normal neurological and neuropsychological examinations. All six are below the maximal age of onset in the family -60 years. This is evidence for low or late penetrance of the AD HSP gene in this family. The identification of normal individuals carrying the disease haplotype demonstrates the importance of genetic studies in combination with clinical examination when counselling at risk family members.
\end{abstract}

Keywords: Hereditary spastic paraparesis; cognitive impairment; SPG4; variable expression; low penetrance; haplotype analysis

Correspondence: Professor Nollaig A . Parfrey, U CD D epartment of Pathology, St. Vincent's H ospital, D ublin 4, I reland. Tel: +353 1 2094784; Fax: +353 1 2837727; E mail: n.parfrey@ucd.ie

R eceived 15 July 1997; revised 27 November 1997; accepted

16 D ecember 1997 


\section{Introduction}

H ereditary spastic paraparesis (H SP) describes a group of clinically and genetically diverse neurodegenerative disorders that are characterised by progressive weakness and spasticity of the lower limbs. Clinically it is classified into 'pure' and 'complicated' forms ${ }^{1}$ according to whether the paraparesis occurs in isolation or with other clinical abnormalities such as mental retardation, ${ }^{2}$ epilepsy, ${ }^{3,4}$ icthyosis ${ }^{5}$ and dementia., ${ }^{6,7}$ Both forms can be inherited in an autosomal dominant (AD), autosomal recessive $(A R)$ or $X$-linked recessive manner.

Both 'pure' and 'complicated' HSP exhibit genetic heterogeneity. Autosomal dominant 'pure' HSP has been linked to three distinct loci on chrosomosome $2 p$ (SPG 4) ${ }^{8,9}$ chromosome $14 q$ (SPG 3$)^{10}$ and chromosome 15p (SPG 6). ${ }^{11}$ To date approximately $45 \%$ of families remain unlinked, indicating the presence of at least one other locus. ${ }^{12}$ Linkage of 'complicated' A D H SP has not been reported to date. Autosomal dominant hyperekplexia associated with spastic paraparesis has been linked to the glycine receptor on chromosome $5 q^{13}$ Mutations in the GLRA1 gene on chromosome 5, which codes for the alpha 1 subunit of the glycine receptor have previously been described for hyperekplexia when it occurs in isolation and also when the hyperekplexia occurs with spastic paraparesis. ${ }^{14} \mathrm{~A}$ locus in the paracentric region of chromosome 8 was linked to 'pure' A R HSP in four out of five Tunisian families examined ${ }^{15}$ providing evidence for genetic heterogeneity in autosomal recessive HSP. Linkage of 'pure' and 'complicated' families to the SPG 1 (X q21-22) and SPG 2 (X q28) loci on the $X$ chromosome confirms the existence of genetic heterogeneity for $X$-linked $\mathrm{HSP}^{16,17}$

Extensive intra- and inter-familial variation is seen in age of symptom onset, rate of disease progression and severity of the disorder. The average age of onset of 'pure' A D H SP is in the second to fourth decade of life. H owever, D ürr et al reported a range from infancy to 63 years within 12 families linked to the SPG 4 locus. ${ }^{18}$ The clinical expression of the disorder within a family includes asymptomatic patients who are unaware of their condition, mildly affected individuals who have spastic gait but are able to walk independently, and severely affected patients who are wheelchair bound. In addition 'complicated' H SP families can show variable expression of other neurological symptoms. G igli et al ${ }^{3}$ reported a family affected with 'complicated' HSP where the additional symptoms of epilepsy and mental retardation were expressed to varying levels in affected family members.

The degree of penetrance of AD HSP has varied between studies and may confuse the issue for counselling. Cooley et $\mathrm{al}^{19}$ reported a family with evidence of a skipped generation and incomplete penetrance. 0 ther authors have indicated that H SP has an age-dependent penetrance that is nearly complete. ${ }^{12}$

We present a family with autosomal dominant HSP and a specific form of cognitive impairment. We provide evidence of linkage to the SPG 4 locus on chromosome 2. This is the first report of clinical heterogeneity at a single locus for autosomal HSP. We present further genetic analysis that indicates late or low penetrance of the HSP gene and highlights features of variable expression. The genetic studies we have performed have identified patients carrying the disease gene who appeared normal at examination. The haplotype analysis of these unaffected individuals demonstrates the importance of performing genetic testing where possible, in addition to neurological examination, in assessing the affection status of at risk patients.

\section{Materials and Methods}

\section{Patients}

We have identified a large I rish pedigree with A D H SP and/or cognitive impairment consisting of 54 living members (Figure 1). For reasons of confidentiality, the pedigree has been disguised. We have examined 44 participating members of this family and six spouses. A II members gave their consent to partake in the study. Two independent examiners performed neurological examination. The criteria used for diagnosis of spastic paraparesis were those proposed by Fink et al. ${ }^{12}$ Neuropsychological (NP) status was assessed using the Cambridge Cognitive Examination (CAMCOG). ${ }^{20}$ The CA M COG has a maximum score of 107. Scores of 80 or less indicate cognitive impairment. In addition a number of family members had extensive NP examination. Nine categories of intellectual functioning were assessed including verbal IQ, performance IQ, verbal memory, visual memory, information processing speed, fine motor speed, verbal fluency, problemsolving ability and perceptual judgement; detailed descriptions of these tests are provided elsewhere. ${ }^{21}$ Failure on more than half of the categories tested was used as a cutoff to identify patients with evidence of cognitive impairment.

\section{DNA Extraction and PCR Amplification}

DNA was extracted from peripheral blood using a standard triton lysis/phenol extraction method. Polymerase chain reactions were carried out in a $10 \mu$ reaction volume using $100 \mathrm{ng}$ genomic DNA as template and $100 \mathrm{ng}$ of each primer. The reactions were carried out in $200 \mu \mathrm{M}$ dN TP, $50 \mathrm{mM} \mathrm{KCl}$, $10 \mathrm{mM}$ Tris- $\mathrm{HCl}$ (pH 9.0), $0.1 \%$ Triton $\mathrm{X}-100,1.5 \mathrm{mM} \mathrm{M} \mathrm{gCl}$ using $0.2 \mathrm{U}$ Taq polymerase. The amplifications were carried 


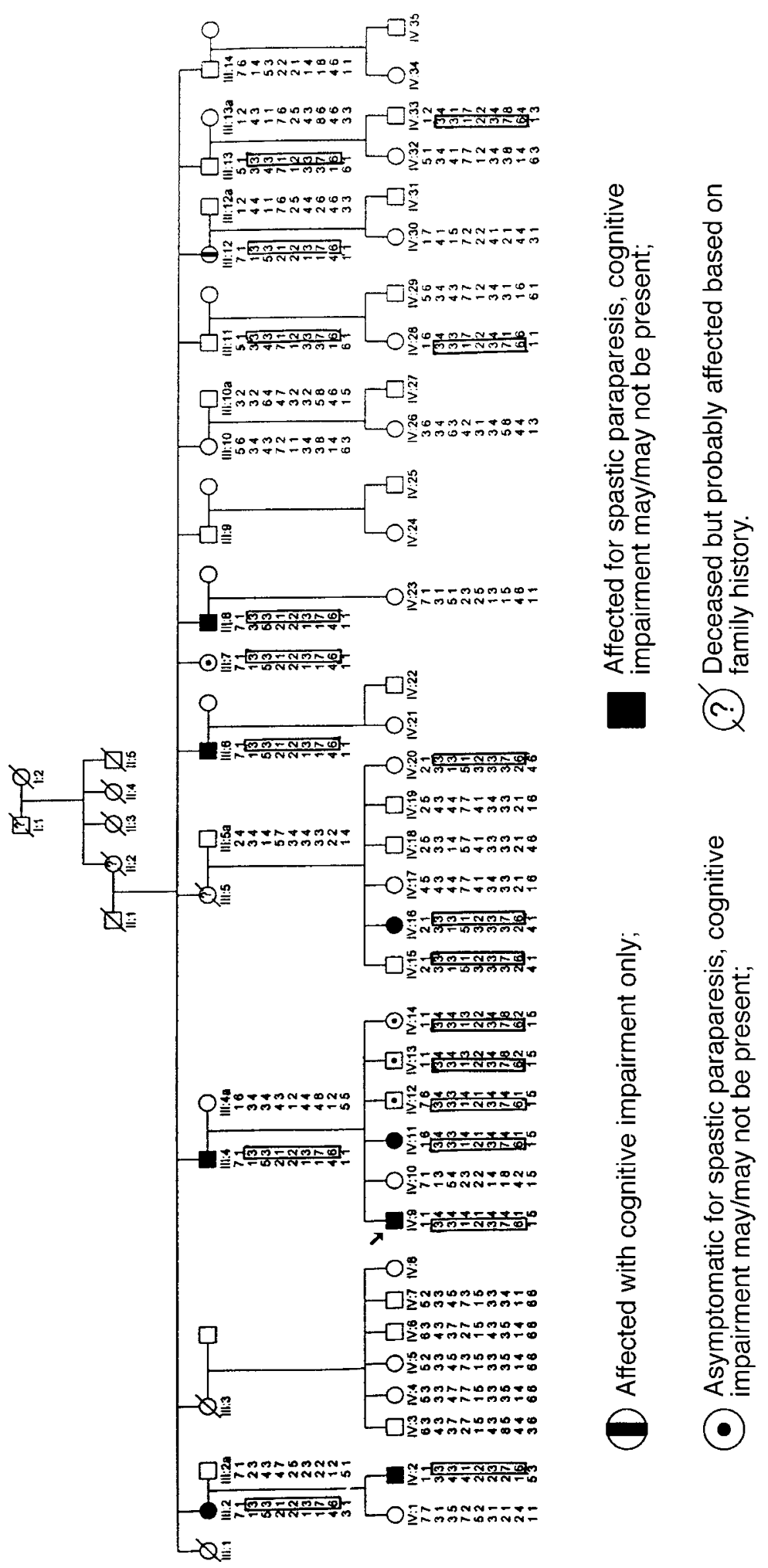

Figure 1 Ped1001: The disease haplotype is boxed. 
out under an overlay of mineral oil on a DNA thermal cycler (Perkin-Elmer Cetus) under the following conditions: $94^{\circ} \mathrm{C}$ $5 \mathrm{~min}$, followed by 25 cycles of $94^{\circ} \mathrm{C} 30 \mathrm{~s}, 55^{\circ} \mathrm{C} 40 \mathrm{~s}$ and $72^{\circ} \mathrm{C}$ $30 \mathrm{~s}, 1$ cycle of $72^{\circ} \mathrm{C}$ for $5 \mathrm{~min}$ and hold at $4^{\circ} \mathrm{C}$. M icrosatellite genotypes were determined for D 14S281, D 14S286, D 14S269, D 15S122, D 15S128, D 15S156, D 15S165, D 2S400, D 2S1325, D 2S2230, D 2S2374, D 2S2331，D 2S2294, D 2S2255, D 2S352, D 2S367 A ND D2S177. U nlabelled amplified products from D 2S400, D 2S1325, D 14S281，D 14S286，D 14S269, D 15S122, D 15S128, D 15S156, and D 15S165 were denatured, electrophoresed on a $6 \%$ denaturing polyacrylamide gel and processed for silver staining using the method of B assam et al. ${ }^{22}$ Primers to amplify remaining microsatellite markers were fluorescently labelled and obtained from PE-A pplied Biosystems, Warrington, UK. In each case, the forward primer was labelled. D2S2230 and D2S2374 with tet, D 2S2331, D 2S2294, D 2S2255, D 2S352 and D 2S367 with fam and D2S177 with hex. The samples were run on A BI 310 PRISM (10s, $15 \mathrm{kV}$ injection; $30 \mathrm{~min}, 15 \mathrm{kV}$ at $42^{\circ} \mathrm{C}$ run). A nalysis performed using G E NE SCA N software was used to assign genotypes for all individuals. The markers used in this study have all been described elsewhere. ${ }^{23,24}$

\section{Linkage Analysis}

The two-point linkage analysis was performed using the subprogram M L I NK of the L I NKA GE (version 5.0) program package ${ }^{25,26}$ as implemented in FA STL INK ${ }^{27}$ assuming equal recombination rates in males and females. H SP was analysed as an autosomal dominant disease with a gene frequency of 0.0001 . To perform the linkage analysis, age-dependent liability classes were determined according to 0 tt et $\mathrm{al}^{28}$ using known age of onset information from affected members in the family. The input for liability classes in LINKAGE was as follows: class 1 ( $0-20$ years) 0 ; class 2 ( $21-25$ years) 0.125 ; class 3 ( $26-36$ years) 0.3125 ; class 4 ( $36-55$ years) 0.5625 and class 5 ( $>56$ years) 0.875 . For each marker, allele frequencies were based on calculated frequencies from unrelated individuals and also from the CEPH database allele frequencies.

\section{Results}

\section{Clinical Data}

There is strong evidence for variable expression within this large nonconsanguineous Irish family (ped1001) affected with A D HSP in association with a late onset specific form of cognitive impairment (Figure 1). The age of onset of spastic paraparesis ranges from 21-60 years (average age 39). Eight of those affected with spastic paraparesis $\left(\mathrm{III}_{2}, \mathrm{II}_{4}, \mathrm{III}_{6}, \mathrm{III}_{8}, \mathrm{IV}_{2}, \mathrm{IV}_{9}, \mathrm{IV}_{11}\right.$, and $\left.\mathrm{IV}_{16}\right)$ had varying degrees of spasticity ranging from moderate (able to walk unaided) to severe (requiring unilateral or bilateral assistance). A further four individuals were asymptomatically affected $\left(\mathrm{III}_{7}\right.$, $I V_{12}, I V_{13}$ and $I V_{14}$ ) and were only diagnosed following abnormal neurological examination (hyperreflexia and extensor plantar responses). Individuals were described as unaffected on the basis of normal neurological examination. All members of the third generation affected with spastic paraparesis also had a specific form of cognitive impairment. $\mathrm{II}_{6}$ was unavailable for evaluation. The cognitive deficit was characterised by impaired performance on visuo-spatial tasks, memory tasks and tasks of fine motor speed and information processing speed. A ffected individuals demonstrated normal performance on tasks of verbal fluency. The cognitive dysfunction manifests itself by difficulty in carrying out new tasks, forgetfulness, poor spatial perception and visuo-motor coordination. 0 ne individual $\left(\mathrm{III}_{12}\right)$ was affected with an identical pattern of cognitive impairment but had normal neurological examination. Individuals affected with cognitive impairment ranged in age from 57-70 years. Neuropsychological evaluation of affected members in the fourth generation did not reveal evidence of this cognitive impairment suggesting a later onset. More detailed clinical and neuropsychological features of this family are described elsewhere. ${ }^{21}$

Individuals III ${ }_{11}, \mathrm{III}_{13}, \mathrm{IV}_{15}, \mathrm{IV}_{20}, \mathrm{IV}_{28}$ and IV $\mathrm{V}_{33}$ were assessed as unaffected following normal neurological examination. Following identification of the disease haplotype in these individuals, their status regarding cognitive impairment was appraised by CA M COG or $\mathrm{NP}$ testing as described. $\mathrm{II}_{11}$ refused to be examined further. III ${ }_{13}$ had a normal CA M CO G test with a score of $92 / 107$. The four members of the fourth generation all had normal CAMCOG scores. Therefore, these haplotype carriers have not yet developed signs of either spastic paraparesis or cognitive impairment.

\section{Linkage of HSP and/or cognitive impairment to chromosome $2 p$}

We have analysed ped1001 for linkage to the known A D HSP loci on chromosomes 2 (SPG 4), 14 (SPG 3) and 15 (SPG 6) using polymorphic microsatellite markers that spanned the candidate regions.

$\mathrm{H}$ aplotype and linkage analysis in a large German pedigree has narrowed the SPG 3 region down to a $7 \mathrm{cM}$ interval between markers D $14 S 288$ and D 14S281. ${ }^{29}$ Linkage of HSP and/or cognitive impairment to this region of chromosome 14 was excluded using D 14S281, D 14S269 and D 14S286. The maximum distance excluded by a single marker was for D14S286 (lod score $=-2.19, \theta=0.07)$. The total distance excluded using these markers spanned the entire SPG 3 region on chromosome 14q.

Linkage to the SPG 6 locus on chromosome 15 was examined using polymorphic microsatellite markers, D 15S122, D 15S128, D 15S156 and D 15S165. The maximum distance excluded for a single marker was $24 \mathrm{cM}$ 
for D 15S128 (lod score $=-2.01, \theta=0.12$ ). Significantly negative lod scores were also obtained for the other markers excluding the entire candidate SPG 6 region. In addition linkage of HSP and associated cognitive impairment to the autosomal recessive locus on chromosome 8 was excluded using D8S279, D8S268, D 8S1113 and D 8S1119 (lod score of -2.08 at $\theta=0.11$ for D 8S279).

Polymorphic markers spanning approximately $20 \mathrm{cM}$ surrounding the candidate SG P4 region were analysed for linkage to HSP and/or cognitive impairment in ped1001. The analysis was performed assuming an agedependent penetrance with liability classes described. Evidence of linkage of HSP and/or cognitive impairment to the SPG 4 locus on chromosome 2 was obtained with a maximum two point lod score of 3.86 at zero recombination for $D 2 S 2374$. For the purposes of linkage analysis all 13 members with spastic paraparesis and/or cognitive impairment were considered affected, including the four asymptomatic affected patients.
Linkage to this region was confirmed by analysis of D 2S2331 $\left(Z_{\max }=3.21\right.$ at zero recombination), and $D 2 S 177$ ( $Z_{\max }=2.99$ at zero recombination). Linkage analysis of D 2S2255, D 2S400, D 2S352, D 2S2230 and D 2S1325 also revealed positive lod scores (Table 1). To allow for the possibility that the family may contain unaffected SPG 4 gene carriers who are not yet expressing signs, a low penetrance, affecteds only analysis was performed. The positive lod scores obtained agree with the evidence of linkage to SPG 4 (Table 1).

\section{Haplotype Analysis}

We have analysed nine polymorphic markers spanning the SPG 4 locus. A nalysis of the resulting genotype data revealed a haplotype that was present in all 12 family members affected with spastic paraparesis. In addition, $\mathrm{III}_{12}$, who has cognitive impairment as the only sign, also has this core haplotype, thus confirming the status as an affected individual. The core haplotype includes a

Table 1 Two point LOD scores following linkage analysis of chromosome $2 p$ polymorphic markers and spastic paraparesis and/or cognitive impairment in ped1001. A ge dependent penetrance was assumed in one analysis (A ge dep. Pen) and an affecteds only analysis in the other

\begin{tabular}{|c|c|c|c|c|c|c|c|c|c|}
\hline \multirow[b]{2}{*}{ M arkers } & \multicolumn{8}{|c|}{ Recombination Fraction $\theta$} & \multirow[b]{2}{*}{0.40} \\
\hline & 0.00 & 0.05 & 0.10 & 0.15 & 0.20 & 0.25 & 0.30 & 0.35 & \\
\hline \multicolumn{10}{|l|}{ D 2S2255 } \\
\hline A ge dep. pen & - & 2.26 & 2.33 & 2.21 & 2.00 & 1.72 & 1.37 & 0.98 & 0.55 \\
\hline $\begin{array}{l}\text { A ffected only } \\
\text { D 2S400 }\end{array}$ & 0.05 & 1.98 & 1.97 & 1.83 & 1.62 & 1.35 & 1.05 & 0.72 & 0.37 \\
\hline A ge dep. pen & 2.43 & 2.34 & 2.18 & 1.97 & 1.72 & 1.43 & 1.11 & 0.77 & 0.41 \\
\hline $\begin{array}{l}\text { A ffected only } \\
\text { D } 2 \text { S352 }\end{array}$ & 2.78 & 2.53 & 2.26 & 1.97 & 1.67 & 1.36 & 1.02 & 0.68 & 0.34 \\
\hline A ge dep. pen & 2.30 & 2.11 & 1.91 & 1.69 & 1.46 & 1.21 & 0.95 & 0.68 & 0.40 \\
\hline $\begin{array}{l}\text { A ffected only } \\
\text { D 2S2374 }\end{array}$ & 1.59 & 1.43 & 1.26 & 1.09 & 0.93 & 0.75 & 0.57 & 0.39 & 0.21 \\
\hline A ge dep. pen & 3.86 & 3.66 & 3.39 & 3.05 & 2.68 & 2.25 & 1.79 & 1.28 & 0.74 \\
\hline $\begin{array}{l}\text { A ffected only } \\
\text { D 2S2230 }\end{array}$ & 3.59 & 3.28 & 2.95 & 2.60 & 2.24 & 1.84 & 1.43 & 0.98 & 0.54 \\
\hline A ge dep. pen & 2.38 & 2.16 & 1.94 & 1.69 & 1.44 & 1.17 & 0.89 & 0.61 & 0.33 \\
\hline $\begin{array}{l}\text { A ffected only } \\
\text { D } 2 S 1325\end{array}$ & 1.77 & 1.59 & 1.41 & 1.22 & 1.03 & 0.83 & 0.62 & 0.41 & 0.21 \\
\hline A ge dep. pen & 2.46 & 2.37 & 2.20 & 1.98 & 1.73 & 1.44 & 1.11 & 0.76 & 0.41 \\
\hline $\begin{array}{l}\text { A ffected only } \\
\text { D 2S177 }\end{array}$ & 2.70 & 2.45 & 2.19 & 1.91 & 1.62 & 1.31 & 0.98 & 0.65 & 0.33 \\
\hline A ge dep. pen & 2.99 & 2.88 & 2.69 & 2.44 & 2.14 & 1.80 & 1.42 & 1.00 & 0.57 \\
\hline $\begin{array}{l}\text { A ffected only } \\
\text { D 2S2331 }\end{array}$ & 3.27 & 2.98 & 2.68 & 2.35 & 2.01 & 1.65 & 1.27 & 0.86 & 0.46 \\
\hline A ge dep. pen & 3.21 & 3.07 & 2.85 & 2.58 & 2.26 & 1.90 & 1.49 & 1.05 & 0.59 \\
\hline $\begin{array}{l}\text { A ffected only } \\
\text { D 2S2294 }\end{array}$ & 3.45 & 3.14 & 2.81 & 2.47 & 2.11 & 1.72 & 1.32 & 0.89 & 0.47 \\
\hline A ge dep. pen & - & 0.44 & 0.67 & 0.72 & 0.69 & 0.61 & 0.49 & 0.34 & 0.19 \\
\hline A ffected only & -1.27 & 0.60 & 0.72 & 0.70 & 0.63 & 0.53 & 0.40 & 0.27 & 0.14 \\
\hline
\end{tabular}


$9 \mathrm{cM}$ region flanked by $\mathrm{D} 2 \mathrm{~S} 400$ and $\mathrm{D} 2 \mathrm{~S} 2331$. R ecombination events in individuals $\mathrm{IV}_{2}$ and IV $\mathrm{V}_{12}$ indicated in Figure 1 have excluded markers D 2S2255 and D 2S2294 from the haplotype. Critical crossover analysis has previously mapped the SPG 4 locus to a $4 \mathrm{cM}$ region flanked by D $2 S 400$ and D 2S367. ${ }^{30}$

A Ithough there is evidence of a skipped generation in ped 1001, close inspection of affected family members indicates an incomplete penetrance. Seven of the fourteen offspring of the third generation were affected with spastic paraparesis and/or cognitive impairment as expected for a completely penetrant autosomal dominant disorder with a segregation ratio of 0.5 . H owever, the penetrance in the fourth generation seems markedly lower with only seven of the 21 at risk individuals showing signs of spastic paraparesis. H aplotype reconstruction was extended to include all participating members of the family. This analysis confirmed the age dependent penetrance of HSP and revealed an additional six unaffected people carrying the disease haplotype.

Two members of the third generation $\left(\mathrm{III}_{11}\right.$ and $\left.\mathrm{III}_{13}\right)$ are carrying the complete haplotype. $\mathrm{III}_{11}$, who had a normal neurological examination at 56 years, has in turn passed the haplotype on to the next generation $\left(I V_{28}\right) . \mid I I_{13}$, aged 55 at examination, had a normal neurological examination and a normal CA M CO G test and is therefore not expressing any signs or symptoms of HSP and/or cognitive impairment. $\mathrm{III}_{13}$ has passed the haplotype on to $\mathrm{IV}_{33}$. Haplotype analysis of members of the fourth generation has identified four unaffected individuals positive for the disease haplotype. Individuals IV ${ }_{15}, \mathrm{IV}_{20}, \mathrm{IV}_{28}$ and $\mathrm{IV}_{33}$ all had a normal neurological and neuropsychological examination at 42, 32, 27 and 23 years, respectively. A Ithough the maximal age of onset of symptoms of spastic paraparesis in ped1001 was 60 years, six of the eight symptomatically affected individuals in ped1001 had onset of symptoms by 40 years. The oldest unaffected member with the disease haplotype to be examined (56 years) has not yet reached the maximal age of onset (60) and so cannot be confidently identified as a case of non-penetrance.

\section{Discussion}

We have presented data providing evidence of linkage of H SP and/or cognitive impairment to the SPG 4 locus on chromosome 2 in ped1001. This is the first evidence of linkage of autosomal 'complicated' H SP to this locus. To date all other families linked to SPG 4 are affected with 'pure' HSP with no other associated signs. This clinical heterogeneity at a single locus has previously been described for $\mathrm{X}$-linked HSP. ${ }^{16,17}$ We have identified a core haplotype of approximately $9.4 \mathrm{cM}$ that segregates with the disease phenotype of HSP and/or cognitive impairment. In addition this haplotype was found to be present in six family members who had normal neurological and neuropsychological examinations. The occurrence of the haplotype in patients affected with varying severity of spasticity and/or cognitive impairment and in individuals as yet unaffected, confirms the variable expression seen in HSP. The unaffected haplotype carriers may indicate nonpenetrant cases of the H SP gene, alternatively they may be due to age dependent penetrance of HSP.

Disease expression varies markedly among carriers of the disease haplotype. Of the 19 carriers, three are affected with spastic paraparesis and cognitive impairment, four with spastic paraparesis only, one with cognitive impairment only, three are asymptomatic for spastic paraparesis, one is asymptomatic for spastic paraparesis but has evidence of cognitive impairment and six appear normal on examination. O ne other, affected with spastic paraparesis $\left(\mathrm{III}_{6}\right)$ was unavailable for neuropsychological testing. Those affected with cognitive impairment are all in the third generation aged 59-72 years. The pattern of cognitive impairment is identical in all five affected members and affects primarily visuo-spatial functions, distinguishing it from common causes for age-related cognitive impairment. $M$ embers of the fourth generation did not have signs of cognitive impairment at examination. However all individuals in the fourth generation are 49 years old or younger and so may develop cognitive impairment at a later stage. Lizcano-Gil et al recently presented two families affected with late onset spastic paraparesis and dementia. ${ }^{6}$ Interestingly, variable expression was not apparent and all individuals developed spastic paraparesis approximately two years prior to the onset of a rapidly progressive dementia characterised by memory loss, emotional changes, judgement disturbances and language alterations.

The identification of the disease haplotype in unaffected individuals demonstrates the importance of genetic studies when examining and counselling families. The occurrence of unaffected disease gene carriers can also complicate linkage studies. A n affected-only 
analysis should be performed when analysing genotype data to exclude any negative contribution made by these unaffected gene carriers. The identification of an H SP haplotype in an unaffected person has previously been reported. Dürr et al $^{18}$ identified a disease haplotype in a single male with a normal neurological examination.

Variable expression is a common feature of autosomal dominant disorders. It has previously been reported in a number of HSP affected families. The molecular basis of variable expression remains to be elucidated. Variable expression has been associated with trinucleotide repeat expansions where the severity of disorder is correlated with the length of expansion. Trinucleotide repeat expansion has recently been reported in chromosome $2 p$-linked families affected with $\mathrm{HSP}^{31}$ Trinucleotide repeat expansion is also associated with the phenomenon of anticipation. E vidence for anticipation has been reported in autosomal dominant 'pure' H SP. ${ }^{30}$ Inspection of ped 1001 indicates a lower age of onset in the third generation, with an average age of onset in the third generation of 49 years compared to average age of onset in the fourth generation of 29 years. There are only three parentoffspring pairs with a known age of onset and although these show a reduction in the age of onset in the fourth generation, the numbers are small and cannot be used as an accurate estimate for anticipation. The occurrence of trinucleotide repeat expansion at the SPG 4 locus raises the possibility of a correlation between age of onset or severity of symptoms with the length of the expansion.

In summary we have linked HSP and/or cognitive impairment to the SPG 4 locus on chromosome 2. We have identified a haplotype segregating with spastic paraparesis and/or cognitive impairment in ped1001. This haplotype was present in all patients regardless of severity. This variable expression may be directly related to the length of triplet repeat expansion. $\mathrm{H}$ aplotype analysis confirmed the status of asymptomatic patients previously diagnosed on the basis of hyperreflexia and extensor plantar response. The disease haplotype was identified in six additional, unaffected patients. This may be due to low penetrance or to late expression of the HSP gene in ped1001. Careful clinical examination of at-risk family members can identify presymptomatic carriers but genetic studies are also required to counsel at-risk patients. In cases where haplotype construction is not possible, caution is advised on counselling unaffected at-risk patients.

\section{Acknowledgements}

This work was supported by a research grant from the $\mathrm{H}$ ealth R esearch Board to P Byrne and N Parfrey.

\section{References}

1 Sutherland J M : Familial spastic paraplegia. $\mathrm{H}$ andbook of Clinical Neurology 1975; 22: 421-431.

2 A llport R B: M ental retardation and spastic paraparesis in four of eight siblings. L ancet II 1971: 1089.

3 Gigli GL, Diomedi M, Bernardi G et al: Spastic paraplegia, epilepsy and mental retardation in several members of a family: A novel genetic disorder. A m J Med Gen 1993; 45: 711-716.

4 Sommerfelt K, Kyllerman M, Sanner G: Hereditary spastic paraplegia with epileptic myoclonus. A cta Neurol Scand 1991; 84: 157-160.

5 Sjörgen T, L arsson T: Oligophrenia in combination with congenital ichthyosis and spastic disorders. A cta P sychiatr Scand 1957; 32: suppl, 1-112.

6 Lizcano-G il LA , G arcia-Cruz D, B ernal-B eltran M, Hernandez $A$ : A ssociation of late onset spastic paraparesis and dementia: Probably an autosomal dominant form of complicated paraplegia. A m J M ed G enet 1997; 68: 1-6.

7 I wabuchi K, Kubota Y, H anihara T, Nagatomo H: Three patients of complicated form autosomal recessive hereditary spastic paraplegia association with hypoplasia of the corpus callosum. No To Shinkei 1994; 46: 941-997.

$8 \mathrm{H}$ azan J, Fontaine B, Bruyn R P et al: Linkage of a new locus for autosomal dominant familial spastic paraplegia to chromosome 2p. H um M ol G enet 1994; 3: 1569-1573.

9 H entati A, Pericak-Vance M A, L ennon F et al: L inkage of a locus for autosomal dominant familial spastic paraplegia to chromosome 2p markers. Hum Mol Genet 1994; 3: 1867-1871.

$10 \mathrm{H}$ azan J, Lamy C, M elki J, Munnich A, de Reconco J, Weissenbach J: A utosomal dominant spastic paraplegia is genetically heterogeneous and one locus maps to chromosome 14q. N at G enet 1993; 5: 163-167.

11 Fink JK, Wu CB, Jones SM et al: Familial spastic paraplegia: Tight linkage to chromosome 15q. A m J H um G enet 1995; 56: 188-192.

12 Fink JK, H einmann-Patterson T: H ereditary spastic paraplegia: A dvances in genetic research. N eurology 1996; 46: 1507-1515.

13 Baxter P, Connolly S, Curtis A et al: Co-dominant inheritance of hyperekplexia and spastic paraparesis. D ev Med Child Neurol 1996; 38: 739-743.

14 EImslie FV, H utchings SM, Spencer $V$ et al: A nalysis of GLRA 1 in hereditary and sporadic hyperekplexia: A novel mutation in a family cosegregating for kyperekplexia and spastic paraparesis. J M ed G enet 1996; 33: 435-436.

15 H entati A, Pericak-Vance M A, H ung W-Y et al: Linkage of 'pure' autosomal recessive familial spastic paraplegia to chromosome 8 markers and evidence of genetic locus heterogeneity. Hum M ol G enet 1994; 3: 1263-1267.

16 Goldblatt J, Ballo R, Sachs B, M oosa A : X-linked spastic paraplegia: Evidence for homogeneity with a variable phenotype. Clin G enet 1989; 35: 116-120. 
17 Bonneau D, R ozet J - M , B ulteau $C$ et al: X-linked spastic paraplegia (SPG 2): Clinical heterogeneity at a single gene locus. J M ed G enet 1993; 30: 381-384.

18 D ürr A, Davoine C-S, Paternotte $C$ et al: Phenotype of autosomal dominant spastic paraplegia linked to chromosome 2. Brain 1996; 119: 1487-1496.

19 Cooley WC, Melkonian G, Moses C, Moeschler JB: A utosomal dominant familial spastic paraplegia: D escription of a large New England family and a study of management. Dev Med \& Child Neurol 1990; 32: 1098-1104.

20 Roth M, Tym E, M ountjoy C: CA M DEX. B r J Psychiatry 1986; 149: 698-709.

21 Webb S, Coleman D, Byrne PC et al: A utosomal dominant hereditary spastic paraparesis with cognitive loss: L inked to chromosome $2 p$. B rain; in press

22 Bassam BJ, Caetano-A nnoles G, G resshoff PM : Fast and sensitive silver staining of DNA in polyacrylamide gels. A nal Biochem 1991; 196: 80-83.

23 D ib C, Fauré S, Samaon D et al: A comprehensive genetic map of the human genome based on 5,264 microsatellites. Nature 1996; 380: 152-154.

24 L evitt R C, K iser M B, D ragwa $C$ et al: Fluorescence-based resource for semiautomated genomic analyses using microsatellite markers. G enomics 1994; 24: 361-365.
25 Lathrop G M, Lalouel J M, Julier J M, Ott J : Strategies for multilocus linkage analysis in humans. PNAS 1984; 81: 3443-3446.

26 Lathrop G M, Lalouel J M, Julier J M, Ott J: Multilocus linkage analysis in humans: Detection of linkage and estimation of recombination. A m J H um G enet 1985; 37: 482-498.

27 Schaffer A A, Gupta K, Shriram K, Cottingham RW: A voiding recomputation in linkage analysis. Hum Hered 1994; 44: 225-237.

$28 \mathrm{Ott}$ J: A nalysis of $\mathrm{H}$ uman $\mathrm{G}$ enetic Linkage. J ohn $\mathrm{H}$ opkins U niversity Press, 1991; pp 155-158.

29 Gispert S, Santos N, D amen R et al: A utosomal dominant familial spastic paraplegia: Reduction of the FSP 1 candidate region on chromosome $14 \mathrm{q}$ to $7 \mathrm{cM}$ and locus heterogeneity. A m J H um G enet 1995; 56: 183-187.

30 Bürger J, M etzke $H$, Paternotte $C$, Schilling $F, H$ azan J, Reis A: Autosomal dominant spastic paraplegia with anticipation maps to a $4 \mathrm{cM}$ interval on chromosome 2p21.p24 in a large German family. H um G enet 1996; 98: 371-375.

31 Nielsen JE, Koefoed $P, A$ bell $K$ et al: CAG repeat expansion in autosomal dominant pure spastic paraplegia linked to chromosome 2p21-p24. H um M ol G en 1997; 6: 1811-1817. 